\title{
Feasibility of Detecting Bioorganic Compounds in Enceladus Plumes with the Enceladus Organic Analyzer
}

\author{
Richard A. Mathies, ${ }^{1}$ Md Enayet Razu, Jungkyu Kim, ${ }^{2}$ Amanda M. Stockton, ${ }^{3}$ Paul Turin, and Anna Butterworth ${ }^{4}$
}

\begin{abstract}
Enceladus presents an excellent opportunity to detect organic molecules that are relevant for habitability as well as bioorganic molecules that provide evidence for extraterrestrial life because Enceladus' plume is composed of material from the subsurface ocean that has a high habitability potential and significant organic content. A primary challenge is to send instruments to Enceladus that can efficiently sample organic molecules in the plume and analyze for the most relevant molecules with the necessary detection limits. To this end, we present the scientific feasibility and engineering design of the Enceladus Organic Analyzer (EOA) that uses a microfluidic capillary electrophoresis system to provide sensitive detection of a wide range of relevant organic molecules, including amines, amino acids, and carboxylic acids, with ppm plume-detection limits (100 pM limits of detection). Importantly, the design of a capture plate that effectively gathers plume ice particles at encounter velocities from $200 \mathrm{~m} / \mathrm{s}$ to $5 \mathrm{~km} / \mathrm{s}$ is described, and the ice particle impact is modeled to demonstrate that material will be efficiently captured without organic decomposition. While the EOA can also operate on a landed mission, the relative technical ease of a fly-by mission to Enceladus, the possibility to nondestructively capture pristine samples from deep within the Enceladus ocean, plus the high sensitivity of the EOA instrument for molecules of bioorganic relevance for life detection argue for the inclusion of EOA on Enceladus missions. Key Words: Labon-a-chip-Organic biomarkers—Life detection-Planetary exploration. Astrobiology 17, 902-912.
\end{abstract}

\section{Introduction}

$\mathbf{E}$ NCELADUS IS AN ICY MOON of Saturn with surface features that range in age from old cratered terrains that formed 4.2-4.6 Ga (Porco et al., 2006; Kirchoff and Schenk, 2009 ) to the south polar terrain (SPT) dominated by prominent tiger stripe troughs that may be younger than $0.5 \mathrm{Ma}$ (Porco et al., 2006). The high albedo of all terrains (Cruikshank et al., 2005) indicates recent global resurfacing with fresh ice, and it was long suspected that material from Enceladus might contribute to the E-ring of Saturn (Pang et al., 1984). In 2006, Cassini confirmed this suspicion by revealing dozens of distinct, narrow geysers erupting thousands of kilometers into space from four warm fractures in the moon's SPT (Porco et al., 2006, 2014; Spahn et al., 2006; Postberg et al., 2011; Mitchell et al., 2015). This plume has a vapor component that is predominantly water with percent levels of $\mathrm{CO}_{2}, \mathrm{~N}_{2}$ or $\mathrm{CO}$ $(m / z=28)$, and $\mathrm{CH}_{4}$, with trace ammonia and larger light organics such as propane (Waite et al., 2006, 2009). The plume also has a solid particulate component that appears to be stratified in particulate size with the largest salty water particulates being near the surface and smaller micron-scale particulates reaching Cassini fly-by altitudes (Schmidt et al., 2008; Hedman et al., 2009; Schneider et al., 2009; Waite et al., 2009; Postberg et al., 2011; Ingersoll and Ewald, 2011). A recent study, combining measurements from Cassini instruments, estimates the plume column density to be about $3 \mu \mathrm{L} / \mathrm{m}^{2}$ ice at $50 \mathrm{~km}$ altitude (Porco et al., 2017, this issue).

Multiple lines of evidence have built a model of Enceladus as having a saline liquid water ocean beneath the icy surface shell that is in direct contact with a rocky core, and that the plume materials are sampled directly from that liquid water ocean (Postberg et al., 2011; Porco et al., 2014;

\footnotetext{
${ }^{1}$ Department of Chemistry, University of California at Berkeley, Berkeley, California.

${ }^{2}$ Department of Mechanical Engineering, Texas Tech University, Lubbock, Texas.

${ }^{3}$ Department of Chemistry and Biochemistry, Georgia Institute of Technology, Atlanta, Georgia.

${ }^{4}$ Berkeley Space Sciences Lab, University of California at Berkeley, Berkeley, California.
}

(C) Richard A. Mathies et al., 2017; Published by Mary Ann Liebert, Inc. This Open Access article is distributed under the terms of the Creative Commons License (http://creativecommons.org/licenses/by/4.0), which permits unrestricted use, distribution, and reproduction in any medium, provided the original work is properly credited. 
Iess et al., 2014; McKinnon 2015; Thomas et al., 2015). Light organics have been directly detected in the plume emanating from this ocean (Waite et al., 2006, 2009), which may mean this subsurface ocean is enriched with complex organics with $m / z$ above the Cassini ion and neutral mass spectrometer (INMS) detection threshold. Indeed, a recent analysis of Cosmic Dust Analyzer data has revealed high mass complex organic cations at the $1 \%$ level in $1-3 \%$ of the ice particles examined (Postberg et al., 2017).

Analysis of a class of Si-containing, $10 \mathrm{~nm}$ scale E-ring particulates indicated ongoing hydrothermal activity at the ocean-core interface (Hsu et al., 2015) similar to that sustained in alkaline hydrothermal vent systems on Earth today. These hydrothermal systems, such as the Lost City system on Earth (Kelley et al., 2005), are alkaline ( $\mathrm{pH} \sim 9-11)$, lowtemperature $\left(\sim 100^{\circ} \mathrm{C}\right)$ systems driven by serpentinization and are very different from magmatically heated hightemperature $\left(\sim 450^{\circ} \mathrm{C}\right)$ acidic on-axis systems. The recent observation of molecular hydrogen in the Enceladus vents provides more evidence for deep ocean hydrothermal processes (Waite et al., 2017). Alkaline hydrothermal systems such as those likely on Enceladus have been proposed on Earth to be suboceanic "hot spots" for the emergence of life on Earth (Russell et al., 2010, 2014). In light of the salty liquid water ocean, laced with organic compounds, exhibiting seafloor hydrothermal activity, and venting kilometers into space, Enceladus is a most promising place in the solar system to conduct astrobiological studies.

The most obvious next step in astrobiological analysis at Enceladus is to determine the organic fraction of its plume with highly sensitive and quantitative compositional analysis. The INMS has detected organic species C2-C5 with individual species abundance $\sim 1$ part per thousand (Waite et al., 2011; McKay et al., 2012), but unfortunately can provide little information about organic species with $\mathrm{m} / \mathrm{z}$ above its mass limit (99 Da) or those that do not produce positive ions with high efficiency (Waite et al., 2004). The total organic fraction of the plume could be as high as that found in primitive carbonaceous chondrites; for example, the Murchison meteorite has $\sim 12 \mathrm{ppm}$ amino acids (Glavin et al., 2010) accumulated through abiotic processes on a primitive parent body that experienced mild heat and liquid water during early solar system formation. Steel et al. (2017, this volume) suggest Enceladus has an environment suitable for formation of amino acids both through abiotic processes, after achieving a steady-state ocean amino acid concentration at $0.1 \mu M$ levels, and based on availability of $\mathrm{H}_{2}$ through serpentinization, they suggest a methanogen-based biotic system that might produce $90 \mu M$ total amino acid ocean concentrations.

Amino acids produced abiotically result in a distribution of racemic (or nearly racemic) amino acid speciation in carbonaceous chondrites that is dependent on the weathering and alteration processes that have impacted the extraterrestrial body (Pizzarello et al., 2012; Pizzarello and Yarnes, 2016). Terrestrial life, however, uses only 20 amino acids in its biopolymers, and thus, the terrestrial biosphere is enriched with a biologically focused distribution of homochiral L-amino acids (Lovelock, 1965; McKay, 2004); sea water on Earth has a total amino acid content of roughly micromolar, with individual amino acid concentrations ranging from 1 to $100 \mathrm{n} M / \mathrm{L}$ (Garrasi et al., 1979). The sensitive, quantitative, compositional, and chiral analysis of amino acids can therefore provide significant information about the multiple processes that have shaped Enceladus, whether they are abiotic or biotic.

To obtain a quantitative, compositional, and chiral analysis of the amino acid composition of the subsurface ocean of Enceladus, multiple requirements must be met. First, the sample must come from that subsurface ocean, which appears to be the case with ice particulate samples of the plume. Capture must occur with high efficiency and with minimal degradation, racemization, and contamination of organic amines and amino acids. Postcapture extraction and analysis must additionally ensure high efficiency with low degradation and racemization; experience with amino acid analysis on Earth and in space has shown that the extraction and analysis systems must both be liquid based. While thermal volatilization gas chromatography/mass spectrometry (GCMS) on Mars, with and without derivatization, has provided information on volatile organic molecules, detection and quantitative compositional analysis of less-volatile oxidized and nitrogen-bearing species of relevance to possible biology-like amino acids remain a challenge for nonliquidbased techniques (Biemann et al., 1976; Stern et al., 2015; Millan et al., 2016). Finally, the detection system must couple the highest possible analytical sensitivity with the liquid-based analysis system. Laser-induced fluorescence (LIF) detection is the most sensitive technique for liquid samples, with concentration limits of detection (LODs) at the detection point as low as 75 pM (Chiesl et al., 2009; Stockton et al., 2010). The mass LODs based on the amount of material in a typical $1 \mathrm{~nL}$ volume CE injection are 300 zeptomoles, vastly outperforming even the most sensitive GCMS femtogram LODs.

Enceladus may be the best possible target in our solar system for detecting organics relevant for biological habitability and bioorganic compounds that are indicative of past or present life. An efficient capture of pristine ice particulates, followed by liquid extraction, a liquid-based separation, and LIF may be the best combination of tools and techniques with which to characterize the organic content of these deep-sea samples. The Enceladus Organic Analyzer (EOA) is an instrument designed by the Berkeley Space Sciences Laboratory (SSL) based on decades of heritage development at the University of California at Berkeley that has all these attributes. The purpose of this article is to present the EOA instrument concept and proof-of-principle laboratory studies, to present the detailed design and engineering development that have been done toward a flyable instrument, and to present modeling studies establishing the feasibility of successfully capturing and analyzing organic molecules from the Enceladus plume in a fly-by mission profile.

\section{General Approach and Heritage}

The EOA technical approach for a fly-by instrument is summarized in Figure 1. The instrument can be configured for a single pass fly-by at up to $5-6 \mathrm{~km} / \mathrm{s}$ or a multiple pass orbiter at lower velocities. The capture door is opened, and the cold $\sim 160 \mathrm{~K}$ exterior capture surface gathers a sample of the Enceladus plume that is $\sim 10 \mathrm{~cm}^{2}$ in cross section and up to $150 \mathrm{~km}$ long. After the encounter, the door is closed and, following warming, water solvent is pumped into the 


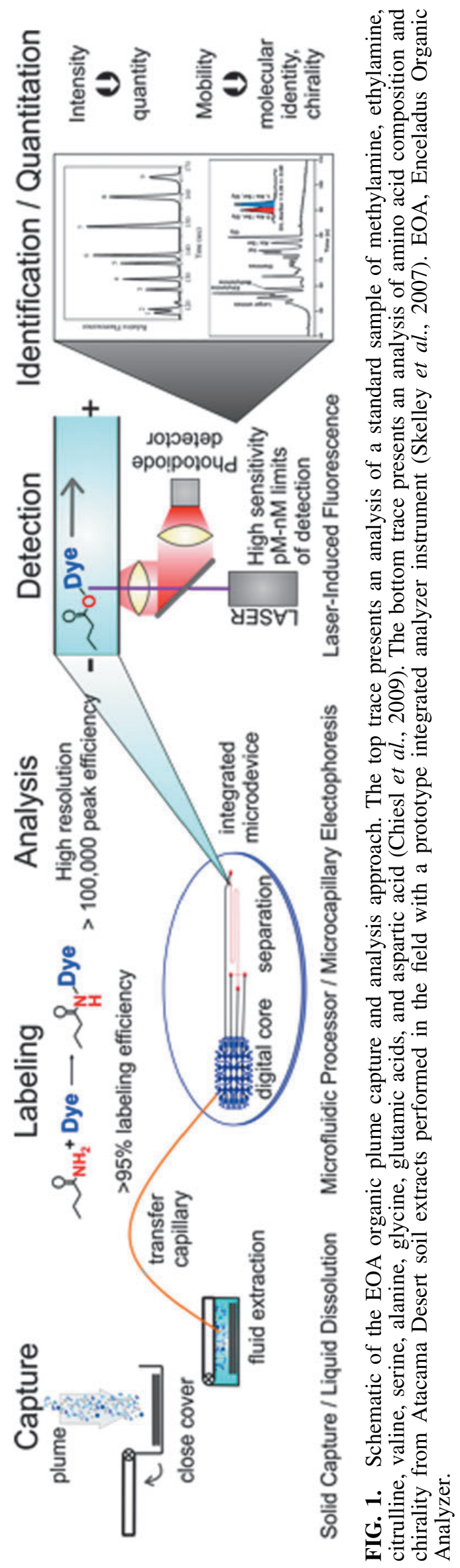

chamber to dissolve the captured organic molecules. The chamber is optimized to maximize the collector area and to minimize depth to keep the water volume for dissolution as low as possible. The sample solution is then pumped back to the microfluidic chip where aliquots are mixed with functional group-specific (amine, carboxylic acid, aldehyde/ ketone, thiol) fluorescent reagents. The labeled samples are then passed to the microfabricated capillary electrophoresis (CE) system, and high voltage is applied to effect highresolution separation followed by LIF detection. The fluorescence intensity provides concentration information, and the time of appearance converted to the intrinsic molecular mobility provides molecular identity. EOA can also perform micellar electrokinetic chromatography (MEKC) with a different buffer to provide chiral resolution of amino acids or other chiral species (Chiesel et al., 2009). In this way, part-per-million detection (in the plume particles themselves) of key amine and amino acid and carboxylic acid molecules that inform habitability and potential for or presence of life is achieved. While this description is focused on plume sampling, the core analyzer is equally well suited to organic analysis on a landed instrument, which would not require the plume capture technology described and modeled in this work. If EOA were placed in the plume snow shadow, an open capture plate could capture orders of magnitude more material (Porco et al., 2017, this issue), resulting in part-per-billion detection capability.

The core technologies in the EOA have been extensively developed at Berkeley through the construction and testing of a wide variety of laboratory-based and field instruments and trials over the past 15 years (for a review see Kim et al., 2016). The first organic instrument was the prototype portable Mars Organic Analyzer (MOA), which integrated a microfluidic CE chip with pneumatic valves for solution control, a high-voltage CE channel, and LIF along with electronics for operation in remote environments (Skelley et al., 2005). The MOA prototype was deployed in field trips to the Panoche Valley, California, as well as the Atacama Desert, Chile (Skelley et al., 2005, 2007), where it exploited its $100 \mathrm{p} M$ concentration limit of detection to detect amino acids at the low ppb levels in the solid samples. A trace from this work is presented in Figure 1. This work provided the basis for its inclusion in the Urey instrument in Phase A advanced development for the ExoMars rover. This Phase A work led to the development of advanced buffer systems to mitigate the effects of high and low $\mathrm{pH}$ samples, high-salt samples, and oxidants (Stockton et al., 2009), low limit of detection labeling and analysis methods with Pacific Blue dye (Chiesl et al., 2009), as well as methods to analyze carboxylic acids, aldehydes, and ketones (Stockton et al., 2010, 2011). This technology also underwent advanced engineering design at the Berkeley SSL for the MOA proposal for Mars 2020.

EOA can analyze for organic amines, carboxylic acids, polycyclic aromatic hydrocarbons (PAHs), etc., to determine organic chemical inventory and likely chemical reactions and processing in the subsurface ocean. Specifically, EOA will analyze for amino acids using amino acid side chain distribution and chirality to probe life indicators and carboxylic acids using chain length variation to probe for biosynthesis (Lovelock, 1965; Balkwill, 1988; McKay, 2004). EOA is significant because its strengths for organic analysis 
(Stockton et al., 2008, 2009, 2010, 2011) complement the weaknesses of existing techniques (Leshin et al., 2013; Ming et al., 2014). The LIF detection system in EOA provides sub-100 p $M$ detection limits in the analyzed solution (Chiesl et al., 2009), and because it is concentration-based rather than mass-based detection it can detect trace $(<\mathrm{ppb})$ species in small micro-to-nanogram input samples (Kaiser et al., 2013). The EOA liquid extraction technique avoids deleterious reactions of organics with oxidants (Stockton et al., 2009) that can compromise thermal volatilization protocols used in GCMS (Freissinet et al., 2015). The EOA prototype was tested on Atacama Desert samples from the Yungay Hills sediments and the Murchison meteorite, and was able to quantitatively determine amino acid composition and chirality from samples (Skelley et al., 2007; Chiesl et al., 2009), where the Sample Analysis at Mars engineering model was unable to detect any organics native to these samples (Stalport et al., 2012). Liquid extraction is also more facile than laser desorption mass spectrometry, which requires that molecules be dissolved in a special matrix with a high laser absorption cross section (Li et al., 2015).

Raman and antibody-based techniques can detect large organic molecules (Parro et al., 2011; Bhartia et al., 2012), but Raman is intrinsically a surface technique for opaque samples with low sensitivity for buried molecules and it requires complex spectral interpretation. Antibody techniques targeted to large, complex biomolecules are Earthcentric approaches (Parro et al., 2011) with nanomolar concentration sensitivity. Antibody techniques that target smaller organic and bioorganic molecules must fortuitously choose the correct targets or develop a very large number of antibody probes. These issues are compounded by the additional difficult requirement for long-duration stabilization of sensitive antibody proteins (Diego-Castilla et al., 2011).

\section{Capture Chamber Design and Rationale}

The tilted louver structure at the input face of the capture chamber is presented in Figure 2. The surfaces that are impacted by the incoming ice particles are fabricated of soft 1100 alloy aluminum to direct the particle kinetic energy into target deformation and to provide efficient thermal transfer of the impact heating into the relatively massive Al surface. These surfaces are also readily cleaned of organic

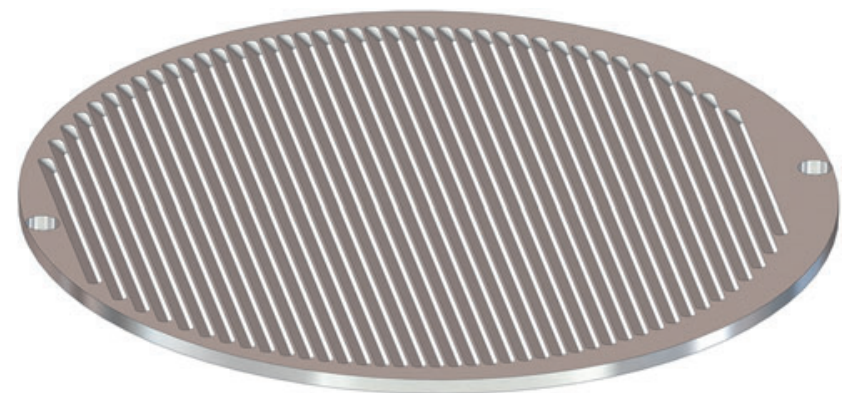

FIG. 2. CAD of the tilted louver particle capture structure in EOA. The louvers are thin $1100 \mathrm{Al}$ slats that capture half of the incoming particles and permit the other half to pass through to the underlying $1100 \mathrm{Al}$ chamber surface. CAD, computer-aided design. residues and contaminants via oxidative pyrolysis and other standard cleaning protocols.

The EOA capture chamber design is justified by the observation that the capture and survival of organic compounds in high-velocity encounters from $200 \mathrm{~m} / \mathrm{s}$ to $6 \mathrm{~km} / \mathrm{s}$ have been demonstrated. U2 aircraft routinely captures interplanetary dust particles with significant organic matter at $200 \mathrm{~m} / \mathrm{s}$ (Brownlee, 1985). Glavin et al. (2008) and Elsila et al. (2009) recovered nanomole amounts of cometary methyl amine, ethyl amine, glycine, and likely $\beta$-alanine from Stardust 1100 foils following $6.1 \mathrm{~km} / \mathrm{s}$ impacts. Burchell et al. (2014) and Bowden et al. (2009) impacted organic-containing ice with projectiles at up to $4.9 \mathrm{~km} / \mathrm{s}$ and demonstrated significant recovery of labile $\beta$-carotene as well as stearic acid and anthracene. Thus, significant data support the survival and capture of organic compounds in hypervelocity impacts with amounts readily detectable by EOA.

In more detail, the capture chamber design consists of a louvered screen placed over a shallow capture chamber with an overall impact area of $10 \mathrm{~cm}^{2}$. The louver screen consists of a parallel array of 100 micron thick louvers fabricated of $1100 \mathrm{Al}$ foil tilted at an angle of $45^{\circ}$ with a size and spacing chosen such that $50 \%$ of the incoming particles strike the louvers and 50\% pass through to the $1100 \mathrm{Al}$ surface below. This design ensures efficient capture of any material that rebounds from the underlying surface impact. The ice particles will strike the cold soft Al surface where the majority of the kinetic energy is deposited into the target in the form of target deformation and heat transfer to the target (Melosh, 1989 and Figure 3).

The kinetic energy that remains will heat and potentially melt the ice. Any material that vaporizes on impact will produce a water organic molecular plume that condenses on the interior of the chamber and the backside of the louvers. Multiple passes can be made to add additional sample, and after closing the collector lid sealing the extraction chamber, the warmed capture plate is washed to remove collected organics for analysis.

If the Enceladus ocean sample harbors extant organisms such as bacteria adapted to extreme environments, impacts in the $2-5 \mathrm{~km} / \mathrm{s}$ range should produce significant shock and/ or heat lysis of cells destroying physical cellular structure but releasing their more stable chemical components and metabolites for chemical analysis. For impacts that do not shatter the cells (Burchell et al., 2001; Horneck et al., 2006), we still expect efficient cell lysis due to the 25 atm difference in osmotic pressure between our water buffer solution and the cell interior, which would be adapted to a very salty Enceladus ocean environment.

A second important consideration is the density of the plume and the amount of material that can be captured in one transit. The plume density estimates vary as a function of plume width, pass altitude, and time. By using the recent estimated plume column density of about $3 \mu \mathrm{L} / \mathrm{m}^{2}$ at $50 \mathrm{~km}$ altitude (Porco et al., 2017, this issue), one transect of a $10 \mathrm{~cm}^{2}$ area capture chamber would thus encounter $3 \mu \mathrm{g}$ ice, which, assuming $1 \mathrm{ppm}$ levels, captures $3 \mathrm{pg}$ of amino acids. Steel et al. (2017, this volume) suggest that amino acids (glycine, alanine, AIB, and glutamic acid) in the ambient Enceladus ocean could total $0.4 \mu M$ due to abiotic production, which corresponds to $\sim 0.1 \mathrm{pg}$ of amino acids in $3 \mu \mathrm{g}$ 
captured ice. Their modeled methanogenic amino acid production result was $90 \mu M$ ocean and plume amino acid concentration, which would result in $20 \mathrm{pg}$ total amino acids collected in a single $10 \mathrm{~cm}^{2}$ transect. A $90 \mu \mathrm{M}$ collected sample is equivalent to a $2000 \mathrm{p} M \mathrm{CE}$ injection, which is easily detectable given the high sensitivity of the EOA instrument.

\section{Numerical Analysis of Ice Particle Impact Temperature}

The ice particle impact temperature profile is important for determining the ice capture efficiency as well as the likelihood of organic analyte survival. From conservation of momentum and energy as shown in Equations 1 and 2 in the Supplementary Data (Supplementary Data are available online at www.liebertonline.com/ast), the change in kinetic energy of the incoming ice plume due to foil impact results in heating of the ice and the collection chamber foil, deformation of the foil, and vibration of the chamber. In this modeling, we make a worst-case assumption that $50 \%$ of the kinetic energy is converted to heating of the captured material and the soft Al 1100 target (Melosh, 1989). Numerical analysis indicates only a negligible increase $(0.25 \mathrm{~K})$ in the final temperature of the collected ice plume and chamber due to plume impact for the collection of $4.4 \mu \mathrm{g}$ ice corresponding to the unmasked capture chamber diameter and $100 \mathrm{~km}$ plume width for an altitude of $50 \mathrm{~km}$ as shown in Figure $3 \mathrm{~A}$ calculated from Equation 3 in the Supplementary Data.

The maximum temperature of the ice and foil after the impact was calculated for conductive heat transfer and the combination of impact and conduction from Equation 5 in the Supplementary Data under the assumption of minimum radiative heat gain/loss from space to the foil for the period of ice particle collection (Fig. 3B). The maximum
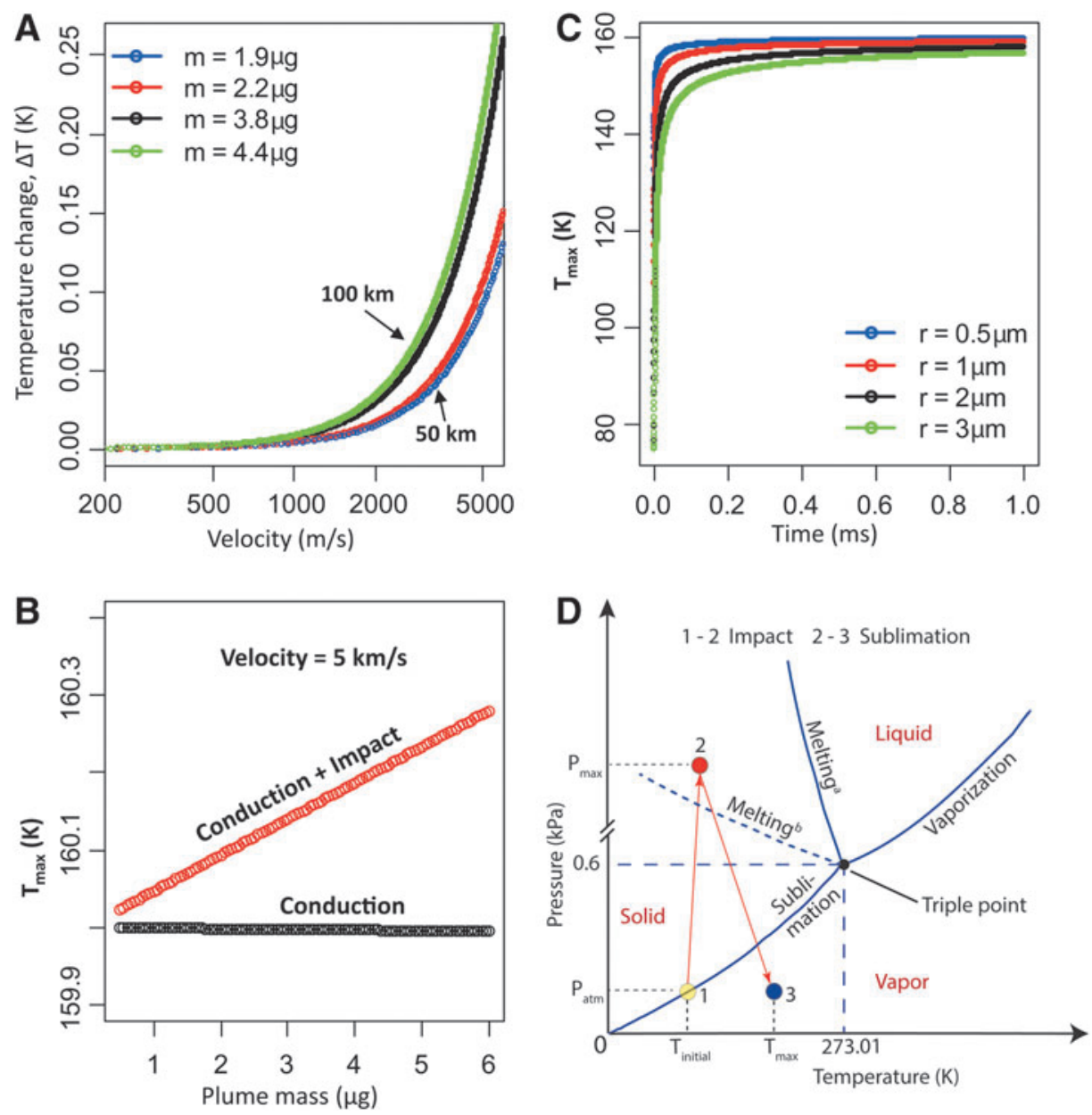

FIG. 3. Theoretical modeling of high-velocity ice particle collection on $1100 \mathrm{Al}$ foil. (A) Profile of the temperature change $(\Delta \mathrm{T})$ in the captured ice and foil due to impact of four different total captured plume masses. Plume mass was calculated for an altitude of $50 \mathrm{~km}$ for $50 \mathrm{~km}$ and $100 \mathrm{~km}$ plume width from Visual and Infrared Mapping Spectrometer data for both masked and unmasked capture chamber diameter as shown in Supplementary Data. (B) Maximum temperature of the ice plume and fly-by capture chamber at $5 \mathrm{~km} / \mathrm{s}$ impact velocity as a function of plume mass for conduction only and for the combined conduction and impact case, which is slightly above the foil temperature. (C) Transient temperature profile of individual ice particles versus time after impact using transient heat conduction for semi-infinite solid as in Equation 4 in Supplementary Data. (D) PressureTemperature $(\mathrm{P}-\mathrm{T})$ diagram for water illustrating the possible thermodynamic state of collected ice based on temperature analysis in (A-C) and the pressure in space and pressure after impact for an individual particle using Hooke's law and the elastic modulus of $1100 \mathrm{Al}$. Minimum pressure $\left(\mathrm{P}_{\min }\right)$ is unknown, $\mathrm{P}_{\max }$ is $40 \mathrm{GPa}, \mathrm{T}_{\text {initial }}$ is $75 \mathrm{~K}$, and $\mathrm{T}_{\max }$ is $160.2 \mathrm{~K}$. Yellow, red, and blue dots show the three states of the ice particle-initial, after impact, and before closing the door, respectively. The liquidsolid coexistence curve is indicated under static pressure conditions (Melting ${ }^{\mathrm{a}}$ ) and also shock pressure conditions (Melting ${ }^{\mathrm{b}}$ ). 
temperature remains in a range of $159.98-160.25 \mathrm{~K}$, which is close to the initial temperature of the foil with negligible increase due to impact for the range of plume mass captured.

The surface temporal profile of the ice projectile temperature during impact was also calculated for individual impacting particles of $0.5,1,2$, and $3 \mu \mathrm{m}$ radius using Equation 4 in the Supplementary Data (Fig. 3C). The temperature of the ice particle exponentially increases to 120 $140 \mathrm{~K}$ in a few nanoseconds, while asymptotically reaching the maximum contact surface temperature $(160.25 \mathrm{~K})$ in $100 \mathrm{~ns}$ to $1 \mathrm{~ms}$ with slight variation for varying particle sizes $(0.5-3 \mu \mathrm{m})$, respectively. With this temperature profile, no thermal degradation of entrained organics is expected.

A phase change of the ice particle from solid to liquid and especially vaporization should be avoided for efficient capture of organic molecules in the incoming ice. The phase change depends on the temperature and pressure of the ice particle during and after impact; it is desirable to remain to the left and above the triple point in Figure 3D $(0.6 \mathrm{kPa}, 273.01 \mathrm{~K})$. Since the Enceladus plume consists of a mixture of ice particles and vapor, there is a possibility that the initial thermodynamic state of the captured ice particles is a quasiequilibrium between the water ice and vapor. During the impact, the pressure and temperature in the ice particle increase momentarily (1-2 in Fig. 3D) and then the pressure starts to drop, whereas temperature keeps increasing up to $162.25 \mathrm{~K}$. At the final state indicated by 3 in Figure 3D, there is likely sublimation of the water in the captured ice due to elevated temperature and low pressure before the capture door is closed.

\section{EOA Design Schematics and Operation}

A computer-aided design of the entire EOA instrument along with a mask design for the microchip analyzer is pre- sented in Figure 4. The overall instrument is a compact $2.5 \mathrm{~kg}$ in a $16 \times 16 \times 12 \mathrm{~cm}$ package with low 2-3 watt survival power requirements to stay between $-10^{\circ} \mathrm{C}$ and $-50^{\circ} \mathrm{C}$. The capture chamber design, operation, and functional parameters and an overview of EOA operation were already described. Below, we detail the operational process and parameters.

Macroscopic fluid transport between major components of the instrument such as the extractant chamber, capture chamber, and waste reservoirs is driven by applying gas pressure through a stainless steel bellows system. All macroscopic volumes of fluids are contained within a welded stainless steel bellows that can be expanded or contracted by externally applied gas pressure. This approach avoids the problem of gas-fluid bubbles in large containers under microgravity.

Fluidic transport in microchannels is driven by pneumatic peristaltic pumps fabricated within the chip itself via typically three polydimethylsiloxane (PDMS) membrane valves placed in series (Grover et al., 2003). In the 50-500 micron features within the chip, water surface tension is the dominant force compared to gravity, and pumped liquids reliably sweep bubbles out of the microfluidic channels. These pumps and channels work well right side up $(+1 \mathrm{~g}$ force) and upside down $(-1 \mathrm{~g}$ force $)$ and so reliable operation in microgravity is anticipated (Stockton et al., 2016). PDMS is stable well outside the anticipated operational parameters, and its decomposition products include glass and other species that do not interfere with EOA analyses.

Figure 4 presents the design of the $100 \mathrm{~mm}$ diameter PMA microdevice showing both the PDMS multilayer pneumatic features (blue), PDMS fluidic channels (black), and the glass features (red) in projection. The features on the perimeter indicate where the 48 pneumatic inputs pass into
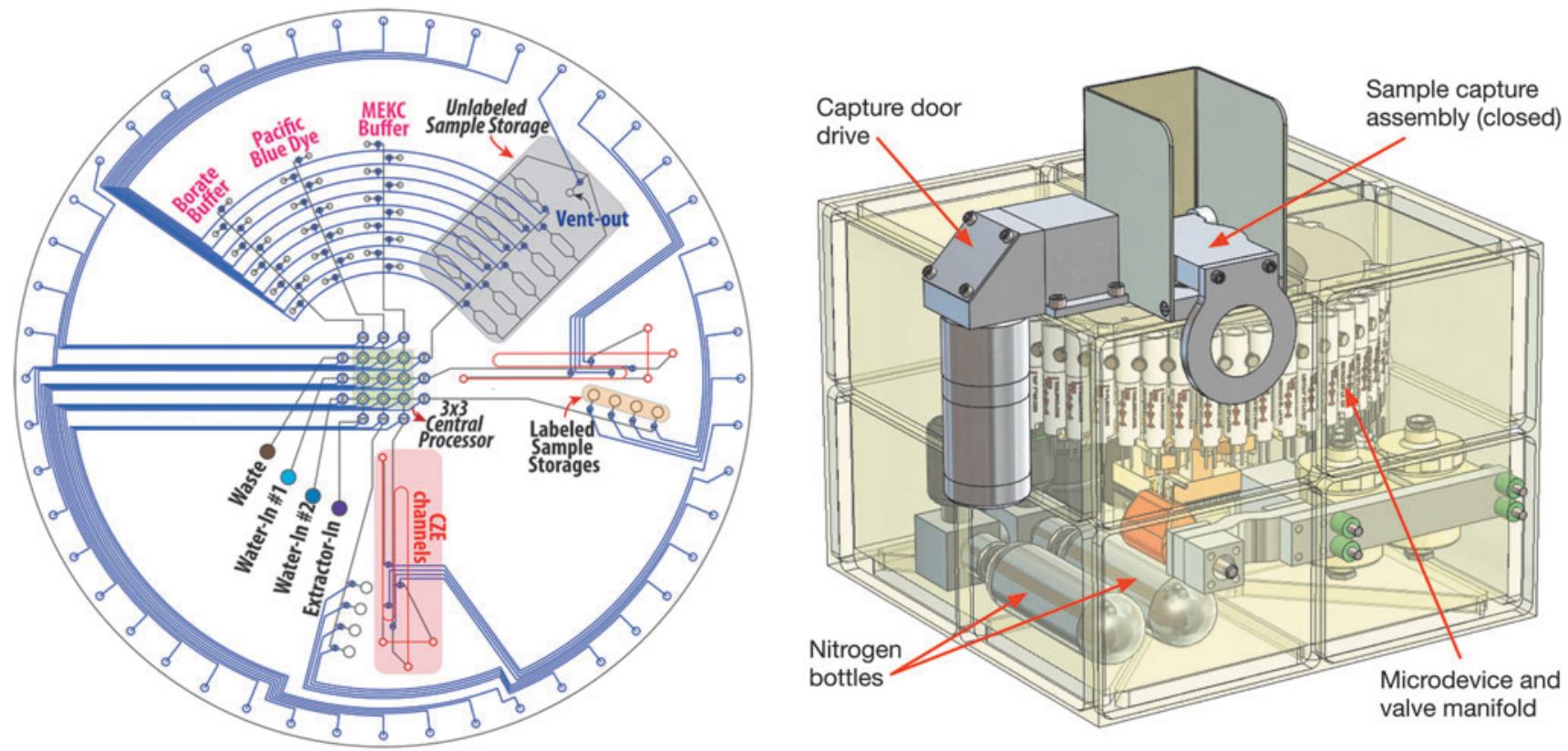

FIG. 4. CAD of the EOA chip fluidic processor and integrated instrument. The TRL of the microfluidic valve array, the microfluidic CE system, and the LIF system is 5; the TRL of the capture plate is currently 3 so this will be the primary focus of development work in the near future along with subsystem integration. CE, capillary electrophoresis; LIF, laser-induced fluorescence; MEKC, micellar electrokinetic chromatography; TRL, technology readiness level. 
the PMA from the manifold solenoids to control fluidic processing by valve actuation. The two $30 \times 100$ micron by $10 \mathrm{~cm}$ long glass CE channels are fabricated within a thermally fused glass sandwich structure drilled via holes that fluidically connect the four ends of the CE system with the PDMS fluidic layers. The central core consists of a $3 \times 3$ valve array processor with each perimeter valve of the array controlled by a gate valve. Each leg of the CE system (sample, waste, cathode, and anode) is connected to the central processor by a fluidic channel that is controlled to route fluid to the desired $\mathrm{CE}$ leg. Two redundant $\mathrm{CE}$ systems are presented in this design.

There are four primary inputs to the PMA that connect with the capture chamber, the two primary solvent reservoirs, and the waste. The 90 reagents and buffers needed for 8 assay series are placed dry in the radial features at the top of the chip. These chambers are addressed by a binary coding format where a reagent is selectively hydrated by pneumatically addressing one of the circumferential lines while pumping water up a selected radial from the $3 \times 3$ processor. In this way, only 19 control valves are needed to address 90 different reagent reservoirs.

To perform an analysis, the closed capture chamber containing sample is warmed and buffer is transported into the chamber dissolving the sample. The dissolved sample is then transported from the capture reservoir to the storage location on the chip (Kim et al., 2016). The amine labeling and analysis are representative; analogous methods for analyzing organic acids (Stockton et al., 2011), aldehydes and ketones (Stockton et al., 2010), thiols (Mora et al., 2013), and PAHs (Stockton et al., 2008) have been presented. For amine analysis, water is first pumped to one of the dry Pacific Blue storage reservoirs to dissolve and mobilize the labeling reagent, and water is pumped to one of the $\mathrm{pH} 9.5$ borate storage reservoirs to hydrate the labeling buffer. Then, alternating $500 \mathrm{~nL}$ aliquots of the $\mathrm{Pa}$ cific blue sample and labeling buffer are pumped by the processor to one of the labeled sample storage locations for a 5-10 min incubation. The $\mathrm{pH} 9.5$ buffer is pumped to the CE reservoirs where the channels fill rapidly by capillary action. The reacted sample is then diluted with separation buffer and pumped to the CE reservoir for injection and analysis. To perform additional separations of similarly sized amines and for chiral analysis of amino acids, the stored Pacific Blue-labeled sample is mixed with the hydrated CHAPSO (3-([3-cholamidopropyl] dimethylammonio)-2-hydroxy-1propane sulfonate) detergent buffer by the PMA and processed for chiral MEKC analysis (Chiesl et al., 2009). After separation, the fluid in the CE reservoirs is pumped out by the processor and sent to waste, and the channel is washed to remove residual buffer components.

The electrophoretic analysis process consists of loading the sample into the CE cross by applying $500 \mathrm{~V} / \mathrm{cm}$ from sample to waste for $30 \mathrm{~s}$. Then, a field of $600 \mathrm{~V} / \mathrm{cm}$ is applied to the separation channel inducing electro-osmotic flow. The molecules in the sample are swept by the electro-osmotic flow toward the detector but also experience a charge-based force and a size-based drag, providing the unique molecular charge and size information that determine identity. Typical separations take $<5 \mathrm{~min}$. If analyte concentrations are high, digital dilutions can be performed in the PMA followed by reanalysis. If the sample concentrations are low, a stack or direct injection can be performed for $2-10 \mathrm{~s}$ to increase the amount of sample injected by more than 10 -fold and extend the detection limits. The detection system will use a $405 \mathrm{~nm}$ diode laser focused to a 30 micron spot within the $\mathrm{CE}$ channel and emission gathered by a high numerical aperture lens and passed through a long-pass filter and confocal filter to a photodiode.

With the optimized dye Pacific Blue, the detection limit for normal injection is $75 \mathrm{p} M$ (Chiesl, 2009), but this limit can be improved $\sim 10$-fold by stack injection to $10 \mathrm{pM}$. This is equivalent to the detection of only attomoles of sample material. A typical example of a CE analysis trace of labeled amines from Chiesl et al. (2009) is presented in Figure 5 .

The analysis process and expected performance of the EOA are outlined in Figure 5. Based on the $75 \mathrm{p} M$ limit of detection at the LIF detector, the EOA should be able to detect as little as $100 \mathrm{p} M$ amino acid, which translates to only 0.4 ppm amino acid (only $1 \mathrm{pg}$ ) in an assumed $3 \mu \mathrm{g}$ of captured ice. The EOA picomolar limit of detection and microfluidic fluid handling enables the split of the $100 \mu \mathrm{L}$ concentrated sample into multiple aliquots while maintaining concentrations at a high enough ratio to the limit of detection to enable quantitation. This LOD is much better than the nanomolar LODs of the best antibody-based analysis systems. In addition, EOA can perform spiking experiments of known standards to reduce ambiguity of identification, since each analysis requires in total only a few microliters of labeled sample solution. While the radiation resistance of the Pacific Blue and Cascade Blue fluorescent probes and of the buffers used by EOA has not yet been explicitly determined, similar fluorescent probes, including fluorescein, SYBR Green, and Alexa Fluor 633, all show more than sufficient stability for a mission profile like that of the EOA (Thompson et al., 2006; Le Postollec et al., 2009; de Diego-Castilla et al., 2011; Carr et al., 2013). Furthermore, the dyes and buffers are stored dry, cold, and in an inert atmosphere on-chip to minimize chemical degradation.

We also consider the performance of the EOA instrument if the amount of ice captured is $<3 \mu \mathrm{g}$. For example, Porco et al. have estimated that the plume ice density may range from 0.2 to $6 \mu \mathrm{L} / \mathrm{m}^{2}$ (Porco et al., 2017, this issue), resulting in the capture of $0.2-6 \mu \mathrm{g}$ of ice by our system; achieving a ppm limit of detection could be challenging if $<1 \mu \mathrm{g}$ ice is captured. One solution to this problem is to perform multiple passes through the plume with an orbiter at a lower altitude to gather more material. In this circumstance, the capture chamber door is open for each pass, and the sample is integrated on the cold capture chamber surface before analysis. An additional way to get more sample would be to shift to a landed configuration and gather plume "snow" with an open capture chamber (Porco et al., 2017, this issue). Second, given that the solution volume returned from the capture chamber $(1000 \mu \mathrm{L})$ is much more than that needed for microfluidic analysis, additional concentration ( $\sim 10$-fold) can be performed onchip to compensate for the reduced sample mass. Third, we can perform stack injection on the $\mathrm{CE}$ system to decrease the limit of detection by a factor of 10 and accept a modest loss of resolution to maintain the ppm limit of detection. Thus, the EOA has the capability to detect ppm organic molecules even if the plume density is at its lower estimated limits. 

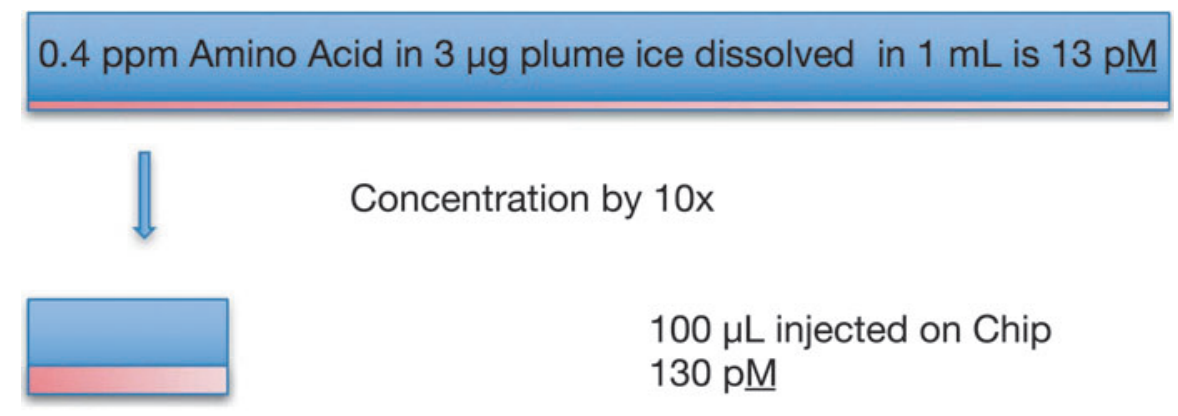

$100 \mu \mathrm{L}$ injected on Chip

FIG. 5. Outline of transfer process for the serial solution transfers in the EOA. Based on the $75 \mathrm{p} M$ limit of detection at the LIF detector, the EOA should be able to detect as little as $100 \mathrm{p} M$ amino acid, which translates to only $0.4 \mathrm{ppm}$ amino acid in an assumed $3 \mu \mathrm{g}$ of captured ice. Thus, the EOA has the capability to detect the capture of as little as $1 \mathrm{pg}$ of an organic species in the capture chamber. The bottom trace presents a microchip CE analysis of a complex mixture of organic amines and amino acids demonstrating the highresolution rapid separations with $75 \mathrm{p} M$ limits of detection that are achieved with Pacific Blue labeling (Chiesl et al., 2009).
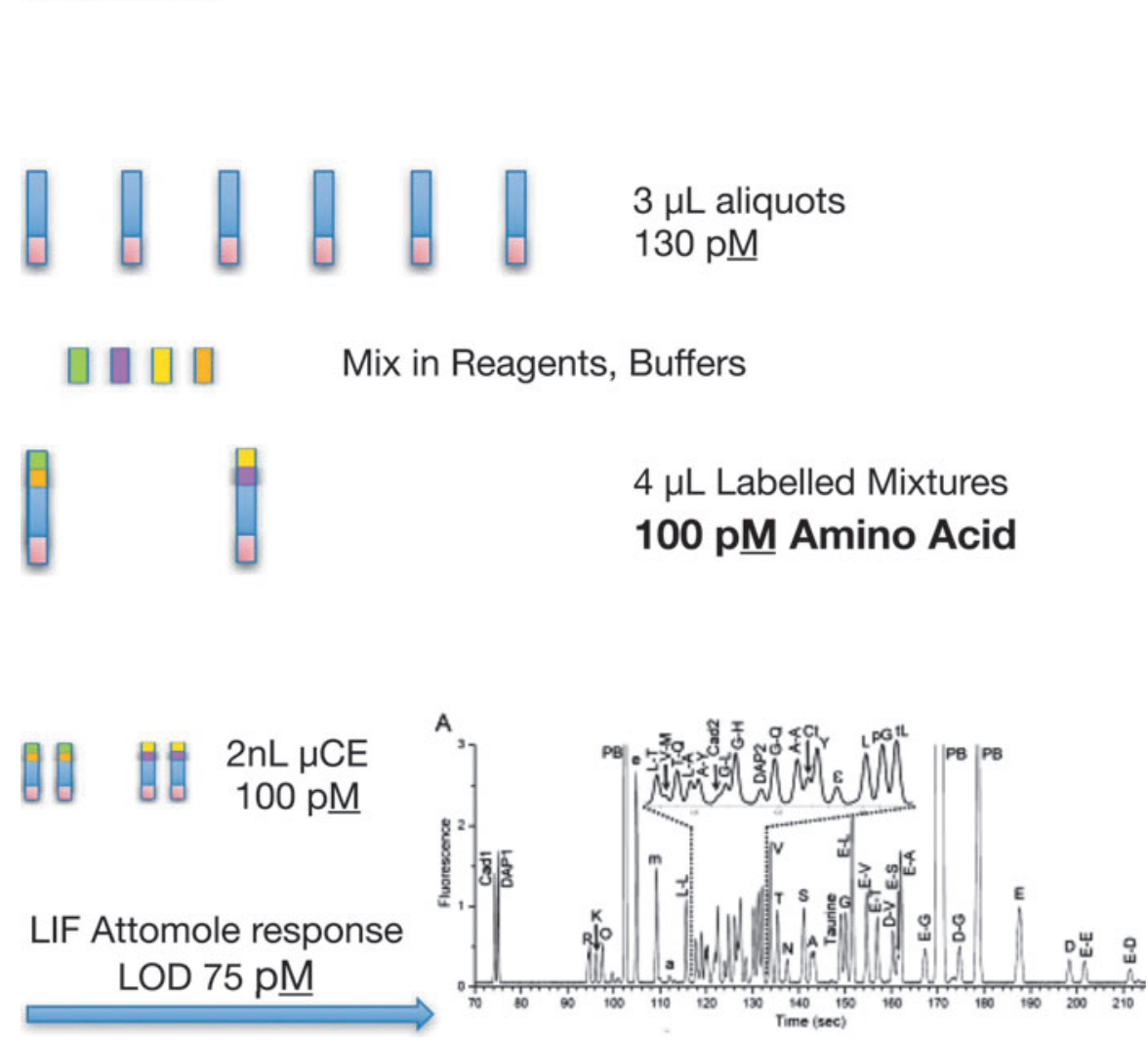

\section{Discussion and Conclusions}

We have presented the concept as well as a practical design for the EOA that can gather ice particles from an Enceladus plume and sensitively analyze for organic molecules, especially organic amines and amino acids that are indicative of habitability and potentially life. In addition, calculations are presented for the amount of ice that can be captured in a single plume transect based on latest available plume density estimates, and the impact is modeled to determine the nature of the impact and the maximum sample temperature during capture. Enceladus plume density understanding is still in flux, but EOA can detect specific organic species at the ppm level from only micrograms of captured ice because of its picogram organic mass detection limits. The organic content in the buried ocean may be lower than parts per million; for example, the $0.1 \mu M(0.01 \mathrm{ppm})$ abiotic amino acid oceanic concentration suggested by Steel et al. (2017) would result in EOA collection of a $30 \mathrm{fg}$ amino acid sample. However, formation of the plume itself induces bubble scrubbing of organics off surfaces that can enhance the organic content by factors of 100-1000 (Porco et al., 2017, this volume). This enhancement would allow even the lowest expected amino acid concentration to be analyzed by EOA.

Thermal modeling shows that the ice particles that impact the soft $1100 \mathrm{Al}$ target with a velocity from $200 \mathrm{~m} / \mathrm{s}$ to $5 \mathrm{~km} / \mathrm{s}$ melt as a molecular solid in static pressure (Melting ${ }^{\mathrm{a}}$ in Fig. 3D) and as a liquid in shock pressure (Melting ${ }^{\mathrm{b}}$ in Fig. 3D), and their temperature rises to the ambient temperature of the target $160 \mathrm{~K}$ on a millisecond time scale (Stewart and Ahrens, 2003; Schwegler et al., 2008). This modest heating is due to the small mass-driven kinetic energy of the incoming particles and the rapid thermal equilibration between the ice particle (with a high surface to volume ratio) and the aluminum surface in which it is imbedded. This thermal profile supports the efficient capture of incoming ice particles because they do not melt or vaporize on impact. This profile also supports the idea that any organic molecules in the ice will not be degraded on impact and that any amino acids or other bioorganics will not be isomerized or racemized thereby preserving composition and chirality as a probe for extraterrestrial life.

The environmental challenges associated with flying a microfluidic system in space are also clearly surmountable. Multilayer microfluidic chips with valves were first made in 
2003 and found to survive many thousands of cycles (Grover et al., 2003). Chips made for the MOA in 2005 were recently examined and found to be fully functional after 10 years of storage (Duca et al., 2015; Stockton et al., 2016). Thus, the microfluidic systems and valve arrays are quite robust. Because the instrument is designed to house the microdevice in a gas-pressurized chamber, operation of the instrument in the vacuum of space should not present a problem. Microfluidic operation in a microgravity environment is also not expected to present an issue because surface tension of fluids in the internal micron-sized channels is the dominant force that controls fluid behavior. Bubbles should not form in the microfluidic systems because of the high surface tension of water, but if formed do not significantly impact performance (Stockton et al., 2016) and can be eliminated by the use of degassing membranes. Finally, fluidic leaks and contamination are unlikely because the entire microfluidic system is monolithically integrated (as opposed to troublesome tubing systems assembled from components); furthermore, if leaks do occur, the volumes of material are very small-on the microliter scale. We conclude that microfluidics presents a uniquely capable approach for performing complex processing of liquid samples for many types of analyses in flight applications.

Once developed, EOA will provide an important expansion of National Aeronautics and Space Administration (NASA) technical capability for the analysis of plume, surface, or subsurface samples from Enceladus, Europa, Titan, or comets. While landed missions generally sample more material, one significant advantage of the fly-by configuration is that the EOA instrument does not require stringent planetary protection. Such missions with EOA technical capability should answer key Decadal Survey questions such as "are there modern habitats elsewhere in the solar system with necessary conditions, organic matter, water, energy, and nutrients to sustain life, and do organisms live there now?"

\section{Acknowledgments}

The authors thank Michael Ludlam and Carolyn Porco for many valuable discussions and contributions. J.K. thanks Texas Tech University for financial support of chip design and ice capture modeling through new investigator start-up funding. A.S. and J.K. additionally acknowledge support by NASA under grant NNX15AM98G. A.B. and P.T. thank University of California Berkeley Space Sciences Lab for support of this project.

\section{Author Disclosure Statement}

No competing financial interests exist.

\section{References}

Balkwill, D.L., Leach, F.R., Wilson, J.T., McNabb, J.F., and White, D.C. (1988) Equivalence of microbial biomass measures based on membrane lipid and cell wall components, adenosine triphosphate and direct counts in subsurface aquifer sediments. Microb Ecol 16:73-84.

Bhartia, R., Hug, W.F., Reid, R., and Salas, E.C. (2012) Noncontact, reagentless, nondestructive, detection of organics, biosignatures, and water. In Proceedings SPIE 8385, Sensors and Systems for Space Applications V, 83850E, edited by K.D. Pham, J.L. Cox, R.T. Howard, H. Zmuda, pp 1-9.
Biemann, K., Oro, J., Toulmin, P., III, Orgel, L.E., Nier, A.O., Anderson, D.M., Simmonds, P.G., Flory, D., Diaz, A.V., Rushneck, D.R., and Biller, J.A. (1976) Search for organic and volatile inorganic compounds in two surface samples from the Chryse Planitia region of Mars. Science 194:72-76.

Bowden, S.A., Parnell, J., and Burchell, M.J. (2009) Survival of organic compounds in ejecta from hypervelocity impacts on ice. Int J Astrobiology 8:19-25.

Brownlee, D.E. (1985) Cosmic dust: collection and research. Annu Rev Earth Planet Sci 13:147-173.

Burchell, M.J., Bowden, S.A., Cole, M., Price, M.C., and Parnell, J. (2014) Survival of organic materials in hypervelocity impacts of ice on sand, ice, and water in the laboratory. Astrobiology 14:473-485.

Burchell, M.J., Mann, J., Bunch, A.W., and Brandão, P.F.B. (2001) Survivability of bacteria in hypervelocity impact. Icarus 154:545-547.

Carr, C.E., Rowedder, H., Vafadari, C., Lui, C.S., Cascio, E., Zuber, M.T., and Ruvkun, G. (2013) Radiation resistance of biological reagents for in situ life detection. Astrobiology 13:6878.

Chiesl, T.N., Chu, W.K., Stockton, A.M., Amashukeli, X., Grunthaner, F., and Mathies, R.A. (2009) Enhanced amine and amino acid analysis using Pacific Blue and the Mars Organic Analyzer microchip capillary electrophoresis system. Anal Chem 81:2537-2544.

Cruikshank, D.P., Owen, T.C., Ore, C.D., Geballe, T.R., Roush, T.L., de Bergh, C., Sandford, S.A., Poulet, F., Benedix, G.K., and Emery, J.P. (2005) A spectroscopic study of the surfaces of Saturn's large satellites: $\mathrm{H}_{2} \mathrm{O}$ ice, tholins, and minor constituents. Icarus 175:268-283.

De Diego-Castilla, G., Crus-Gil, P., Mateo-Marti, E., FernandezCalvo, P., Rivas, L.A., and Parro, V. (2011) Assessing antibody microarrays for space missions: effect of long-term storage, gamma radiation, and temperature shifts on printed and fluorescently labeled antibodies. Astrobiology 11:759-773.

Duca, Z.A., Tan, G.K., Cantrell, T.P., Van Enige, M.A., Mathies, R.A., and Stockton, A.M. (2015) Demonstration of the longevity of microdevices for in situ analysis of organic molecules on outer planet icy moons. In European Planetary Science Congress, September 27-October 2, Nantes, France.

Elsila, J.E., Glavin, D.P., and Dworkin, J.P. (2009) Cometary glycine detected in samples returned by Stardust. Meteorit Planet Sci 44:1323-1330.

Freissinet, C., Glavin, D.P., Mahaffy, P.R., et al. (2015) Organic molecules in the Sheepbed Mudstone, Gale Crater, Mars. J Geophys Res Planets 120:495-514.

Garrasi, G., Degens E.T., and Mopper, K. (1979) The free amino acid composition of seawater obtained without desalting and preconcentration. Mar Chem 8:71-85.

Glavin, D.P., Callahan, M.P., Dworkin, J.P., and Elsila, J.E. (2010) The effects of parent body processes on amino acids in carbonaceous chondrites. Meteorit Planet Sci 45:1948-1972.

Glavin, D.P., Dworkin, J.P., and Sandford S.A. (2008) Detection of cometary amines in samples returned by Stardust. Meteorit Planet Sci 43:399-413.

Grover, W.H., Skelley, A.M., Liu, C.N., Lagally, E.T., and Mathies, R.A. (2003) Monolithic membrane valves and diaphragm pumps for practical large-scale integration into glass microfluidic devices. Sens Actuator B Chem 89:315-323.

Hedman, M.M., Nicholson, P.D., Showalter, M.R., Brown, R.H., Buratti, B.J., and Clark, R.N. (2009) Spectral observations of the Enceladus plume with Cassini-VIMS. Astrophys $J$ 693:1749. 
Horneck, G. (2006) Bacterial spores survive simulated meteorite impact. In Biological Processes Associated with Impact Events, edited by C. Cockell, I. Gilmour, and C. Koeberl, Springer Berlin Heidelberg, pp. 41-53.

Hsu, H.W., Postberg, F., Sekine, Y., Shibuya, T., Kempf, S., Horanyi, M., Juhasz, A., Altobelli, N., Suzuki, K., Masaki, Y., Kuwatani, T., Tachibana, S., Sirono, S., Moragas-Klostermeyer, G., and Srama, R. (2015) Ongoing hydrothermal activities within Enceladus. Nature 519:207-210.

Iess, L., Stevenson, D.J., Parisi, M., Hemingway, D., Jacobson, R.A., Lunine, J.I., Nimmo, F., Armstrong, J.W., Asmar, S.W., Ducci, M., and Tortora, P. (2014) The gravity field and interior structure of Enceladus. Science 344:78-80.

Ingersoll, A.P., and Ewald, S.P. (2011) Total particulate mass in Enceladus plumes and mass of Saturn's E ring inferred from Cassini ISS images. Icarus 216:492-506.

Kaiser, R.I., Stockton, A.M., Kim, Y.S., Jensen, E.C., and Mathies, R.A. (2013) On the formation of dipeptides in interstellar model ices. Astrophys $J$ 765:111.

Kelley, D.S., Karson, J.A., Fruh-Green, G.L., Yoerger, D.R., Shank, T.M., Butterfield, D.A., Hayes, J.M., Schrenk, M.O., Olson, E.J., Proskurowski, G., Jakuba, M., Bradley, A., Larson, B., Ludwig, K., Glickson, D., Buckman, K., Bradley, A.S., Brazelton, W.J., Roe, K., Elend, M.J., Delacour, A., Bernasconi, S.M., Lilley, M.D., Baross, J.A., Summons, R.T., and Sylva, S.P. (2005) A serpentinite-hosted ecosystem: the Lost City hydrothermal field. Science 307:1428-1434.

Kim, J., Stockton, A.M., Jensen, E.C., and Mathies, R.A. (2016). Pneumatically actuated microvalve circuits for programmable automation of chemical and biochemical analysis. Lab Chip 16:812-819.

Kirchoff, M.R., and Schenk, P. (2009) Crater modification and geologic activity in Enceladus' heavily cratered plains: Evidence from the impact crater distribution. Icarus 202:656-668.

Le Postollec, A., Coussot, G., Baque, M., Incerti, S., Desvignes, I., Moretto, P., Dobrijevic, M., and Vandenbeele-Trambouse, O. (2009) Investigation of neutron radiation effects on polyclonal antibodies (IgG) and fluorescein dye for astrobiological applications. Astrobiology 9:637-645.

Leshin, L.A., Mahaffy, P.R., Webster, C.R., et al. (2013) Volatile, isotope, and organic analysis of Martian fines with the Mars Curiosity Rover. Science 341:1238937.

Li, X., Danell, R.M., Brinckerhoff, W.B., Pinnick, V.T., van Amerom, F., Arevalo, R.D., Getty, S.A., Mahaffy, P.R., Steininger, H., and Goesmann, F. (2015) Detection of trace organics in Mars analog samples containing perchlorate by laser desorption/ionization mass spectrometry. Astrobiology 15:104-110.

Lovelock, J.E.A. (1965) A physical basis for life detection experiments. Nature 207:568-570.

McKay, C.P. (2004) What is life and how do we search for it in other worlds? PLoS Biol 2:e302.

McKay, C.P., Khare, B.N., Amin, R., Klasson, M., and Kral, T.A. (2012) Possible sources for methane and C2-C5 organics in the plume of Enceladus. Planet Space Sci 71:73-79.

McKinnon, W.B. (2015) Effect of Enceladus' rapid synchronous spin on interpretation of Cassini gravity. Geophys Res Lett 42:2137-2143.

Melosh, H.J. (1989) Impact Cratering: A Geologic Process, Oxford Monographs on Geology and Geophysics, Clarendon Press, Oxford.

Millan, M., Szopa, C., Buch, A., Coll, P., Glavin, D.P., Freissinet, C., Navarro-Gonzalez, R., Francois, P., Coscia, D., Bonnet, J.Y., Teinturier, S., Cabane, M., and Mahaffy, P.R. (2016)
In situ analysis of martian regolith with the SAM experiment during the first mars year of the MSL mission: Identification of organic molecules by gas chromatography from laboratory measurements. Planet Space Sci 129:88-102.

Ming, D.W., Archer, P.D., Glavin, D.P., et al. (2014) Volatile and organic compositions of sedimentary rocks in Yellowknife Bay, Gale Crater, Mars. Science 343:1245267.

Mitchell, C., Porco, C., and Weiss, J. (2015) Tracking the geysers of Enceladus into Saturn's E ring. Astron J 149:156.

Mora, M.F., Stockton, A.M., and Willis, P.A. (2013) Analysis of thiols by microchip capillary electrophoresis for in situ planetary investigations. Electrophoresis 34:309-316.

Pang, K.D., Voge, C.C., Rhoads, J.W., and Ajello, J.M. (1984) The E-Ring of Saturn and satellite Enceladus. J Geophys Res 89:9459-9470.

Parro, V., de Diego-Castilla, G., Rodriguez-Manfredi, J.A., Rivas, L.A., Blanco-Lovex, Y., Sebastian, E., Romeral, J., Compostizo, C., Herrero, P.S., Carcia-Marin, A., Moreno-Paz, M., GarciaVilladangos, M., Cruz-Gil, P., Peinado, V., Martin-Soler, J., PerezMercader, J., and Gomez-Elvira, J. (2011) SOLID3: a multiplex antibody microarray-based optical sensor instrument for in situ life detection in planetary exploration. Astrobiology 11:15-28.

Pizzarello, S., Schrader, D.L., Monroe, A.A., and Lauretta, D.S. (2012) Large enantiomeric excesses in primitive meteorites and the diverse effects of water in cosmochemical evolution. Proc Natl Acad Sci U S A 109:11949-11954.

Pizzarello, S., and Yarnes, C.T. (2016) Enantiomeric excesses of chiral amines in ammonia-rich carbonaceous meteorites. Earth Planet Sci Lett 443:176-184.

Porco, C., DiNino, D., and Nimmo, F. (2014) How the geysers, tidal stresses, and thermal emissions across the south polar terrain of Enceladus are related. Astron J 148:45.

Porco, C.C., Dones, L., and Mitchell, C. (2017) Could it be snowing microbes on Enceladus? Assessing conditions in its plume and implications for future missions. Astrobiology 17:876-901.

Porco, C.C., Helfenstein, P., Thomas, P.C., Ingersoll, A.P., Wisdom, J., West, R., Neukum, G., Denk, T., Wagner, R., Roatsch, T., Kieffer, S., Turtle, E., McEwen, A., Johnson, T.V., Rathbun, J., Veverka, J., Wilson, D., Perry, J., Spitale, J., Brahic, A., Burns, J.A., DelGenio, A.D., Dones, L., Murray, C.D., and Squyres, S. (2006) Cassini observes the active south pole of Enceladus. Science 311:1393-1401.

Postberg, F., Khawaja, N., Kempf, S., Waite, J.H., Glein, C., Hus, H.W., Srama, R. (2017) Complex organic macromolecular compounds in ice grains from Enceladus [abstract no 1401]. In Lunar and Planetary Science Conference LPS XLVII, Lunar and Planetary Institute, Houston.

Postberg, F., Schmidt, J, Hillier, J., Kempf, S., and Srama, R. (2011) A salt-water reservoir as the source of a compositionally stratified plume on Enceladus. Nature 474:620.

Russell, M.J., Barge, L.M., Bhartia, R., Bocanegra, D., Bracher, P.J., Branscomb, E., Kidd, R., McGlynn, S., Meier, D.H., Nitschke, W., Shibuya, T., Vance, S., White, L., and Kanik, I. (2014) The drive to life on wet and icy worlds. Astrobiology 14:308-343.

Russell, M.J., Hall, A.J., and Martin, W. (2010) Serpentenization as a source of energy at the origin of life. Geobiology 8:355-371.

Schmidt, J., Brilliantov, N., Spahn, F., and Kempf, S. (2008) Slow dust in Enceladus' plume from condensation and wall collisions in tiger stripe fractures. Nature 451:685-688.

Schneider, N.M., Burger, M.H., Schaller, E.L., Brown, M.E., Johnson, R.E., Kargel, J.S., Dougherty, M.K., and Achilleos, N.A. (2009) No sodium in the vapour plumes of Enceladus. Nature 459:1098-1101. 
Schwegler, E., Sharma, M., Gygi, F., and Galli, G. (2008) Melting of ice under pressure. Proc Natl Acad Sci US A 105:14779-14783.

Skelley, A.M., Aubrey, A.D., Willis, P.A., Amashukeli, X., Ehrenfreund, P., Bada, J.L., Grunthaner, F.J., and Mathies, R.A. (2007) Organic amine biomarker detection in the Yungay region of the Atacama Desert with the Urey Instrument. $J$ Geophys Res 112:G04S11.

Skelley, A.M., Scherer, J.R., Aubrey, A.D., Grover, W.H., Ivester, R.H.C., Ehrenfreund, P., Grunthaner, F.G., Bada, J.L., and Mathies, R.A. (2005) Development and evaluation of a microdevice for amino acid biomarker detection and analysis on Mars. Proc Natl Acad Sci U S A 102:1041-1046.

Spahn, F., Schmidt, J., Albers, N., Horning, M., Makuch, M., Seiss, M., Kempf, S., Srama, R., Dikarev, V., Helfert, S., Moragas-Klostermeyer, G., Krivov, A.V., Sremcevic, M., Tuzzolino, A.J., Economou, T., and Grun, E. (2006) Cassini dust measurements at Enceladus and implications for the origin of the E-ring. Science 311:1416-1418.

Stalport, F., Glavin, D.P., Eigenbrode, J.L., Bish, D., Blake, D., Coll, P., Szopa, C., Buch, A., McAdam, A., Dworkin, J.P., and Mahaffy, P.R. (2012) The influence of mineralogy on recovering organic acids from Mars analogue materials using the "one-pot" derivatization experiment on the Sample Analysis at Mars (SAM) instrument suite. Planet Space Sci 67:1-13.

Steel, E.L., Davila, A., and McKay, C.P. (2017) Abiotic and biotic formation of amino acids in the Enceladus Ocean. Astrobiology 17:862-875.

Stern, J.C., Sutter, B., Freissinet, C., Navarro-Gonzalez, R., McKay, C.P., Archer, P.D., Buch, A., Brunner, A.E., Coll, P., Eigenbrode, J.L., Fairen, A.G., Franz, H.B., Glavin, D.P., Kashyap, S., McAdam, A.C., Ming, D.W., Steele, A., Szopa, C., Wray, J.J., Martin-Torres, F.J., Zorzano, M.P., Conrad, P.G., and Mahaffy, P.R. (2015) Evidence for indigenous nitrogen in sedimentary and aeolian deposits from the Curiosity rover investigations at Gale crater, Mars. Proc Natl Acad Sci U S A 112:4245-4250.

Stewart, S.T. and Ahrens, T.J. (2003) Shock Hugoniot of $\mathrm{H}_{2} \mathrm{O}$ ice. Geophys Res Lett 30:1332.

Stockton, A.M., Chiesl, T.N., Scherer, J.R., and Mathies, R.A. (2008) Polycyclic aromatic hydrocarbon analysis with the Mars Organic Analyzer microchip capillary electrophoresis system. Anal Chem 81:790-796.

Stockton, A.M., Chiesl, T.N., Lowenstein, T.K., Amashekuli, X., Grunthaner, F.G., and Mathies, R.A. (2009) Capillary electrophoresis analysis of organic amines and amino acids in saline and acidic samples using the Mars Organic Analyzer. Astrobiology 9:823-830.

Stockton, A.M., Duca, Z., Cato, M., Cantrell, T., Foreman, S., Kim, J., Putman, P., and Schmidt, B. (2016) An Organic Analyzer Instrument for Highly Sensitive In Situ Organic Detection on an Ice Shell Impact Penetrator Descent Probe. In Third International Meeting on Instrumentation for Planetary Missions, October 24-27, Pasadena, CA.

Stockton, A.M., Tjin, C.C., Chiesl, T.N., and Mathies, R.A. (2011) Analysis of carbonaceous biomarkers with the Mars Organic Analyzer microchip capillary electrophoresis system: carboxylic acids. Astrobiology 11:519-528.

Stockton, A.M., Tjin, C.C., Huang, G.L., Benhabib, M., Chiesl, T.N., and Mathies, R.A. (2010) Analysis of carbonaceous biomarkers with the Mars Organic Analyzer microchip capillary electrophoresis system: aldehydes and ketones. Electrophoresis 31:3642-3649.
Thomas, P., Tajeddine, R., Tiscareno, M., Burns, J., Joseph, J., Loredo, T., Helfenstein, P., and Porco, C. (2016) Enceladus's measured physical libration requires a global subsurface ocean. Icarus 264:37-47.

Thompson, D.P., Wilson, P.K, Sims, M.R., Cullen, D.C., Holt, J.M.C., Parker, D.J., and Smith, M.D. (2006) Preliminary investigation of proton and helium ion radiation effects on fluorescent dyes for use in astrobiology applications. Anal Chem 78:2738-2743.

Waite, J.H., Jr., Combi, M.R., Ip, W.H., Cravens, T.E., McNutt, R.L., Kasprzak, W., Yelle, R., Luhmann, J., Niemann, H., Gell, D., Magee, B., Fletcher, G., Lunine, J., and Tseng, W.L. (2006) Cassini ion and neutral mass spectrometer: Enceladus plume composition and structure. Science 311:1419-1422.

Waite, J.H., Glein, C.R., Perryman, R.S., Teolis, B.D., Magee, B.A., Miller, G., Grimes, J., Perry, M.E., Miller, K.E., Bouquet, A., Lunine, J.I., Brockwell, T., and Bolton, S.J. (2017) Cassini finds molecular hydrogen in the Enceladus plume: Evidence for hydrothermal processes. Science 356:155-159.

Waite, J.H., Jr., Lewis, S., Kasprzak, W.T., Anicich, V.G., Block, B.P., Cravens, T.E., Fletcher, G.G., Ip, W.H., Luhmann, J.G., McNutt, R.L., Niemann, H.B., Parejko, J.K., Richards, J.E., Thorpe, R.L., Walter, E.M., and Yelle, R.V. (2004) The Cassini ion and neutral mass spectrometer (INMS) investigation. Space Sci Rev 114:113-231.

Waite, J.H., Jr., Lewis, W.S., Magee, B.A., Lunine, J.I., McKinnon, W.B., Glein, C.R., Mousis, O., Young, D.T., Brockwell, T., and Westlake, J. (2009) Liquid water on Enceladus from observations of ammonia and ${ }^{40} \mathrm{Ar}$ in the plume. Nature 460:487-490.

Waite, J.H., Jr., Magee, B., Brockwell, T., Zolotov, M.Y., Teolis, B., Lewis, W.S., and the INMS Team. (2011) Enceladus' plume composition. In European Planetary Science Congress Abstracts, Vol. 6, EPSC-DPSC2011-61-4.

Address correspondence to: Richard A. Mathies Department of Chemistry University of California at Berkeley 307 Lewis Hall Berkeley, CA 94720

E-mail: ramathies@berkeley.edu

Submitted 7 February 2017 Accepted 18 June 2017

\begin{aligned} & \multicolumn{1}{c|}{ Abbreviations Used } \\ $\mathrm{CAD} & =$ computer-aided design \\ $\mathrm{CE} & =$ capillary electrophoresis \\ $\mathrm{EOA} & =$ Enceladus Organic Analyzer \\ $\mathrm{GCMS} & =$ gas chromatography/mass spectrometry \\ $\mathrm{INMS} & =$ ion and neutral mass spectrometer \\ $\mathrm{LIF} & =$ laser-induced fluorescence \\ $\mathrm{LODS} & =$ limits of detection \\ $\mathrm{MEKC} & =$ micellar electrokinetic chromatography \\ $\mathrm{MOA} & =$ Mars Organic Analyzer \\ $\mathrm{PAHs} & =$ polycyclic aromatic hydrocarbons \\ $\mathrm{PDMS} & =$ polydimethylsiloxane \\ $\mathrm{SPT} & =$ south polar terrain \\ $\mathrm{SSL} & =$ Space Sciences Laboratory \end{aligned}

